\title{
Comprehensive discovery and characterization of small RNAs in Corynebacterium glutamicum ATCC 13032
}

\author{
Almut Mentz ${ }^{1}$, Armin Neshat ${ }^{1}$, Katharina Pfeifer-Sancar ${ }^{1}$, Alfred Pühler ${ }^{2}$, Christian Rückert ${ }^{1,3}$ and Jörn Kalinowski ${ }^{1,3^{*}}$
}

\begin{abstract}
Background: Recent discoveries on bacterial transcriptomes gave evidence that small RNAs (sRNAs) have important regulatory roles in prokaryotic cells. Modern high-throughput sequencing approaches (RNA-Seq) enable the most detailed view on transcriptomes offering an unmatched comprehensiveness and single-base resolution. Whole transcriptome data obtained by RNA-Seq can be used to detect and characterize all transcript species, including small RNAs. Here, we describe an RNA-Seq approach for comprehensive detection and characterization of small RNAs from Corynebacterium glutamicum, an actinobacterium of high industrial relevance and model organism for medically important Corynebacterianeae, such as C. diphtheriae and Mycobacterium tuberculosis.
\end{abstract}

Results: In our RNA-Seq approach, total RNA from C. glutamicum ATCC 13032 was prepared from cultures grown in minimal medium at exponential growth or challenged by physical (heat shock, cold shock) or by chemical stresses (diamide, $\mathrm{H}_{2} \mathrm{O}_{2}, \mathrm{NaCl}$ ) at this time point. Total RNA samples were pooled and sequencing libraries were prepared from the isolated small RNA fraction. High throughput short read sequencing and mapping yielded over 800 sRNA genes. By determining their 5'- and 3'-ends and inspection of their locations, these potential sRNA genes were classified into UTRs of mRNAs (316), cis-antisense sRNAs (543), and trans-encoded sRNAs (262). For 77 of transencoded sRNAs significant sequence and secondary structure conservation was found by a computational approach using a whole genome alignment with the closely related species C. efficiens YS-314 and C. diphtheriae NCTC 13129. Three selected trans-encoded sRNAs were characterized by Northern blot analysis and stress-specific transcript patterns were found.

Conclusions: The study showed comparable numbers of sRNAs known from genome-wide surveys in other bacteria. In detail, our results give deep insight into the comprehensive equipment of sRNAs in C. glutamicum and provide a sound basis for further studies concerning the functions of these sRNAs.

Keywords: Bacteria, Non-coding RNA, High-throughput sequencing, RNA regulation

\section{Background}

Corynebacterium glutamicum is a non-pathogenic and non-sporulating gram-positive soil bacterium which belongs to the order Actinomycetales. This microorganism has a long history of applications in the production of various amino acids and other industrially relevant compounds [1,2]. Furthermore, it serves as a model

\footnotetext{
* Correspondence: joern.kalinowski@cebitec.uni-bielefeld.de

1 Microbial Genomics and Biotechnology, Center for Biotechnology, Bielefeld

University, Universitätsstraße 27, 33615, Bielefeld, Germany

${ }^{3}$ Technology Platform Genomics, Center for Biotechnology, Bielefeld

University, Universitätsstraße 27, 33615, Bielefeld, Germany

Full list of author information is available at the end of the article
}

organism for close relatives with medical significance such as $C$. diphtheriae or Mycobacterium tuberculosis. The genome sequence established a decade ago $[3,4]$ comprises a circular chromosome with a length of almost 3.3 $\mathrm{Mb}$ and harbors more than 3000 annotated proteincoding sequences (CDS). Based on the complete genome sequence, transcriptional regulation in C. glutamicum has been studied extensively [5] and revealed a complex regulatory network including 97 transcriptional regulator proteins with so far 1443 regulatory interactions [6]. However, only very little is known about small RNAs (sRNA) and their potential regulatory actions in this organism. Information on RNA species 
beside ribosomal RNA (rRNA) or transfer RNA (tRNA) is absent from the current genome annotation. It can only be deduced from the genome sequence that C. glutamicum lacks a sequence homologue of the RNA chaperone Hfq, similar to other Actinomycetales [7]. So far, the only experimentally defined sRNA in C. glutamicum (ArnA) was detected upstream of the GntR-Regulator cg1935 and is located in antisense direction [8].

Recently, regulatory RNAs have been detected in all three domains of life with unexpectedly large numbers, in the range of hundreds per bacterial and thousands per eukaryotic genome. In most cases, these transcripts do not encode proteins and so the term non-coding RNA (ncRNA) is often applied synonymously. All hitherto identified RNA families are collected in the RNAfamilies (Rfam) online database [9], fRNAdb [10], and sRNAdb [11]. Beyond trans-encoded sRNA genes, these databases also include RNA motifs from mRNA leader transcripts of protein-coding genes, some of which regulate translation initiation or cause transcriptional attenuation. Elements such as RNA thermometers are structures sensitive to temperature shifts and control the accessibility of the Shine-Dalgarno sequence of the mRNA leader (reviewed in [12]). The classes of attenuation mechanisms are diverse and include amongst others small molecule-mediated riboswitches (reviewed in [13]) as well as classical attenuators regulated by translation of a small leader peptide.

The length of bacterial sRNAs is generally between 50 and $300 \mathrm{nt}$ [14] and can be up to $500 \mathrm{nt}$ [15]. In addition to RNAs with housekeeping function, in-depth analyses of several sRNAs led to the discovery of various novel regulatory functions. These functions modulate a wide range of responses to stresses and other environmental stimuli (reviewed in [16]) including RNA processing and RNA degradation as well as translation control. Different mechanisms of action have been described, the majority representing interactions through basepairing between sense RNA and regulating antisense RNA. Direct transcriptional regulation through sRNAs seems to occur rarely, and was first discovered for 6S RNA of E. coli [17]. The much more frequent post-transcriptional regulation by trans-encoded sRNAs works through imperfect basepairing with target mRNAs (reviewed in [16]). These sRNAs show stable secondary structures and their genes are generally located in the "intergenic regions" between protein-coding sequences. In contrast, cis-antisense sRNAs (asRNA) genes are located directly in the antisense direction with respect to their target genes and thus show full complementarity (reviewed in [18]).

To date, different strategies have been applied for the systematic genome-wide search for sRNAs. In the enterobacterium $E$. coli, a number of sRNAs have been predicted by computational methods (reviewed in [19]).
Such in silico analyses are usually based on common features of sRNAs such as thermodynamic stability, structure conservation, or sequence similarity between species [20], as well as the existence of Rho-independent terminators at their 3 '-ends [21]. The Rfam database provides sRNA predictions for organisms with known genome sequences calculated from sequence covariance models. In C. glutamicum, four sRNAs are predicted by Rfam, including 6C RNA and the housekeeping RNAs tmRNA, RNAse P, and SRP/4.5S RNA.

Experimental strategies for the discovery of sRNAs in bacteria started with systematic genome-wide screens by shotgun cloning and sequencing of cDNA [22] or by using tiling microarrays (reviewed in [23]) and detected large numbers of sRNAs in all tested organisms. Undoubtedly, new high-throughput sequencing techniques enable the most detailed view on a cellular transcriptome. Thus, RNA-sequencing has emerged as a powerful tool for the detection of bacterial sRNAs [24-26]. The creation of RNA-sequencing libraries can vary between different platforms in high throughput sequencing [27] but there are similarities between the procedures. An important step to increase the coverage of mRNA or sRNA in transcriptome sequencing data is the depletion of highly abundant ribosomal RNAs. Another is to ensure that the strand-information of the RNA is kept in the cDNA sequence. This can be done by using adapters of known sequence to be ligated to the RNA before cDNA synthesis. In addition, various specific enzymatic treatments of the RNA samples can be used for mapping of transcriptional starts [26] or for detection of processing sites [28].

Here, we present the first deep sequencing study of sRNAs in C. glutamicum. Sequencing libraries were created by the "differential" RNA-sequencing (dRNA-Seq) approach [26] with RNA samples from exponential growth phase and stress conditions such as heat and cold shock, salt stress, $\mathrm{H}_{2} \mathrm{O}_{2}$ and diamide stress to gain a broad spectrum of transcription of potential sRNA genes in response to these conditions. Supported by promoter searches, RNA-Seq data were analyzed and led to the detection of novel sRNA genes in C. glutamicum ATCC 13032. In addition, sRNA genes were classified and compared with bioinformatic sRNA predictions based on secondary structure stability and sequence conservation.

\section{Results}

Detection of potential sRNA genes in C. glutamicum ATCC 13032 by transcriptome sequencing and read-mapping

Transcription of sRNAs in bacteria is highly variable under different environmental conditions [16,29]. Hence, for a comprehensive survey of sRNAs in C. glutamicum, we isolated total RNA from C. glutamicum cells grown 
to exponential phase and from cells after a variety of stress treatments and pooled the total RNA samples (Figure 1). The stress treatments were heat shock $\left(50^{\circ} \mathrm{C}\right)$, cold shock $\left(10^{\circ} \mathrm{C}\right)$, oxidative stress $\left(1 \% \mathrm{H}_{2} \mathrm{O}_{2}\right)$, diamide stress $(2 \mathrm{mM})$, and salt stress $(1.5 \mathrm{M} \mathrm{NaCl})$. To enrich small RNA for a transcriptome sequencing (RNA-Seq), the pool of total RNAs was size-selected for transcripts smaller than 250 nucleotides (nt) by precipitation and further depleted of ribosomal RNAs (rRNA) using a hybridization procedure that selectively binds rRNA species with biotinylated probes (Figure 1). The probe:rRNA hybrids were then captured by magnetic beads and removed using a magnet. After this step, the sRNA fraction was split into two samples as in the differential RNA sequencing (dRNA-Seq) approach [26]. Hereby, one sample was treated with a $5^{\prime}$-monophosphate-specific exonuclease which degrades specifically transcripts that are processed or undergoing degradation, thus leaving primary transcripts with native $5^{\prime}$-triphosphate ends. The second sample was left untreated as a representation of the whole small transcriptome of the cell. The small RNA samples were then separately committed to strand-specific sequencing library preparation using the standard Illumina TruSeq Small RNA kit. Both cDNA libraries were sequenced on an Illumina GA IIx sequencer, obtaining 35 bases long single reads from their $5^{\prime}$ ends. Reads were mapped to the chromosome sequence of the C. glutamicum ATCC 13032 wild-type strain [3] using the SARUMAN algorithm implemented in CUDA programming language and run on computer graphics cards [30] allowing for up two mismatches per read. In total, $7,869,859$ reads were uniquely mapped for the primary transcripts sample (library 1; Table 1) and $22,752,379$ reads were uniquely mapped in case of the total small RNA sample (library 2; Table 1). The rRNA was found to be more efficiently depleted in the enzyme-treated library 1 , yielding a proportion of only $3 \%$ of total reads mapping to ribosomal RNA genes. It also became apparent that the enzyme treatment had depleted residual mRNA as seen by the lower fraction of reads attributable to the sense direction of CDS. The remaining set of reads were mapped either cis-antisense to $\mathrm{CDS}$ or to regions with no annotated genome features (potential sRNA fraction). The cis-antisense reads made up 3\% and less in both libraries. Interestingly, the majority of read mappings belong to regions with no annotated features, particularly in the library enriched for primary transcripts (59\%), indicating a high number of potential sRNAs in C. glutamicum.

Characterization of potential sRNA genes with the help of bioinformatic promoter analysis

After filtering of mappings to tRNA and rRNA genes and to putative mRNAs (inside CDS, sense direction),

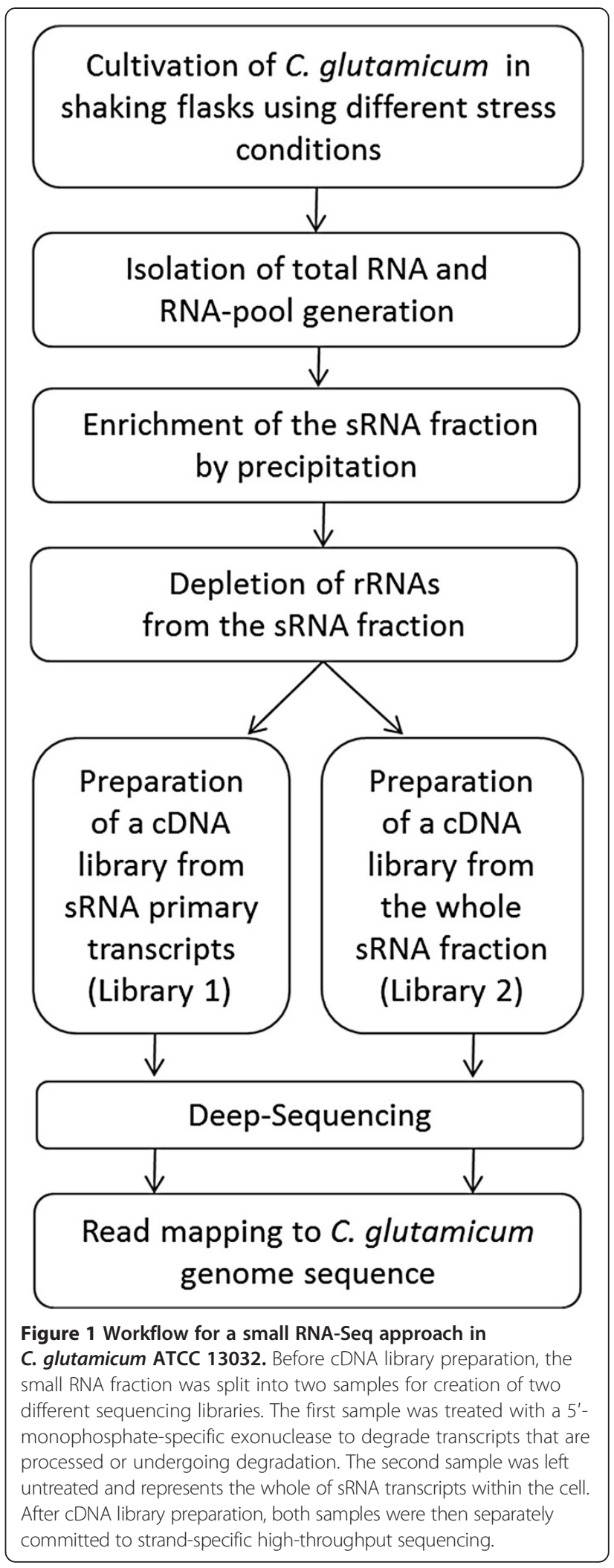


Table 1 Distribution of mapped reads to annotated features in the C. glutamicum 13032 genome in two different sequencing libraries

\begin{tabular}{|c|c|c|c|c|}
\hline \multirow[t]{2}{*}{ Annotated feature category } & \multicolumn{2}{|c|}{ sRNA primary transcripts ${ }^{\mathrm{a}}$ (library 1 ) } & \multicolumn{2}{|c|}{ Whole sRNA transcripts (library 2) } \\
\hline & Reads & [\%] & Reads & [\%] \\
\hline rRNA genes & 255,591 & 3.2 & $5,230,021$ & 23.0 \\
\hline tRNA genes & $1,050,962$ & 13.4 & $2,130,360$ & 9.3 \\
\hline mRNA genes (CDS, sense) & $1,686,575$ & 21.4 & $7,134,344$ & 31.4 \\
\hline \multicolumn{5}{|c|}{ Potential sRNA fraction (remaining set of mapped reads) } \\
\hline cis-antisense (CDS, antisense) & 242,429 & 3.1 & 450,589 & 2.0 \\
\hline Regions with no annotated features & $4,634,302$ & 58.9 & $7,807,065$ & 34.3 \\
\hline Total & $7,869,859$ & & $22,752,379$ & \\
\hline
\end{tabular}

${ }^{\text {a }}$ sRNA primary transcripts were obtained using a 5'-monophosphate-specific exonuclease which degrades specifically transcripts that are either processed or undergoing degradation.

the potential sRNA fraction from the library 1 (primary transcripts enriched) was utilized for the definition of sRNA transcript starts (Figure 2a). A number of reads that start at a distinct genomic position normalized to the previous position was defined as read stack and a transcriptional start site (TSS) was assumed at the 5'position at each of these stacks (Figure 2b). For the experiment performed here, the number of read starts used in stringent filtering was determined to be 20 . This analysis yielded a number of 2899 stacks (1304 stacks cis-antisense to CDS and 1595 stacks in regions lacking annotated features). As a further filtering step, the $5^{\prime}$ - upstream sequences of the assumed TSS were analyzed for promoters. Using the tool Improbizer [31,32], we searched for matches to the consensus promoter sequences recognized by the primary housekeeping sigma factor SigA [33] or the stress-related ECF-family sigma factor SigH [34], which is known to play a major role under oxidative stress [35] and heat stress conditions [36]. Thus, 1267 putative TSS were found to exhibit an upstream SigA-recognized promoter sequence (Figure 2c) (531 cis-antisense to CDS and 736 SigA-dependent promoters in regions lacking annotated features). The search for SigH promoter sequences was successful in (a)

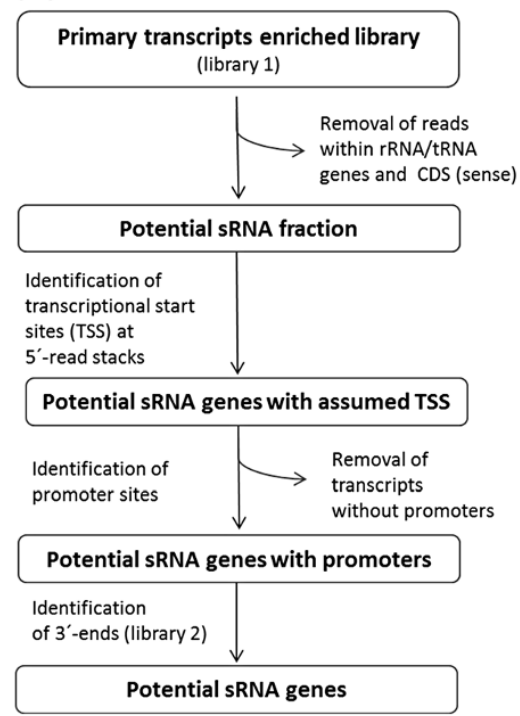

(b)

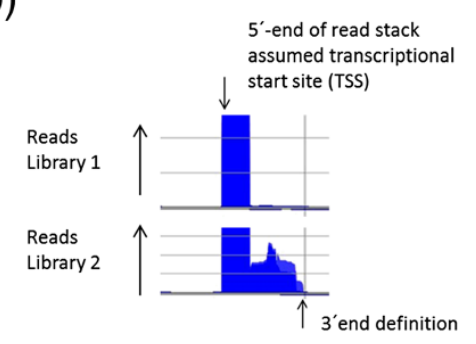

(c)

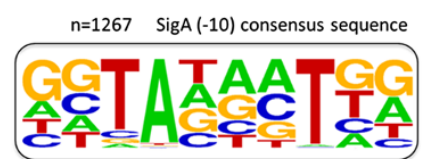

$\mathrm{SigH}(-35)$ consensus sequence $\mathrm{n}=44 \quad \mathrm{SigH}(-10)$ consensus sequence

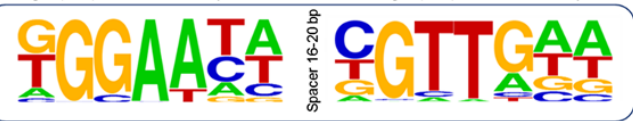

Figure 2 Definition of start and stop positions of potential sRNA genes. (a) Workflow for characterization of sRNA genes with the help of bioinformatic promoter analysis at transcriptional start sites (TSS) in library 1 (primary transcripts enriched). All TSS without promoters were removed and the $3^{\prime}$-ends of potential sRNA genes were determined with sequence data from library 2. (b) Library 1 (primary transcripts enriched) was used for definition of transcriptional start sites at $5^{\prime}$-ends of read stacks. The $5^{\prime}$-end of a read stack is defined as a number of read starts that exceed the number of read starts at the previous position by a factor of 20. (c) Weblogo [37] presentations of the consensus sequences of -35 and -10 core regions. In detail, 1267 SigA and 44 SigH promoter sites were detected by the Improbizer tool. The percentage of occurrence of a nucleotide at a particular position is represented by the size of the nucleotide symbol $(A, C, G, T)$. 
case of 44 TSS (11 cis-antisense to CDS and 33 in regions lacking annotated features). For 14 transcripts, promoters of both types were detected, indicating sRNA variants with different 5 ' -ends.

To determine the 3 '-ends of sRNAs, we mapped reads from the two libraries and followed each of the initial stacks up to a point where the number of read starts fell below the chosen cut-off of 10 reads and defined 3 '-ends from these data (Figure 2c). In addition, we searched for Rho-independent transcription terminators since these have been reported for numerous of bacterial sRNAs [38]. This search was performed with the tool TransTermHP [39] targeting thymine-rich stretches of DNA following a hairpin loop within $60 \mathrm{nt}$ around the assumed 3'ends. Thereby, Rho-independent terminators were found at 69 of these sRNAs (4 cis-antisense to CDS and 65 in regions lacking annotated features).

In 136 cases, the predicted TSS were within close distance to each other, indicating multiple promoters. Proposed multiple starts located within $100 \mathrm{bp}$ at the $5^{\prime}$ end and with the same 3 '-end were merged to a single region and annotated as such.

\section{Classification of potential sRNA genes by their positions relative to annotated protein-coding sequences}

In order to identify putative untranslated regions (UTRs) of mRNAs that are included in the set of potential sRNA genes, these were then grouped according to their position and direction relative to an adjacent CDS (Figure 3). In total, 298 transcripts that had a downstream CDS in less than $100 \mathrm{nt}$ distance from their $3^{\prime}$-ends were designated as "mRNA leader", (Additional file 1). By comparison with the RNA-families database (Rfam [9]) we could additionally assign the well conserved mraW motif (at $c g 2377$ ), the $\operatorname{csp} A$ motif (at $c g 0215$ ), and nine predicted riboswitches which are part of $5^{\prime}$-UTRs longer than 100 nt (Additional file 2). This way we validated the Rfampredicted thiamine pyrophosphate (TPP)-dependent riboswitches upstream of genes from the thiamine biosynthesis pathway, ThiC (cg1476), ThiM (cg1655), and ThiE (cg2236). Two TPP-riboswitches were found upstream of cg0825 (putative beta-ketoacyl acyl carrier protein reductase) and cg1227 (putative membrane protein). One flavin mononucleotide (FMN)-dependent riboswitch was detected at the putative nicotinamide mononucleotide uptake permease (cg0083) and one S-adenosyl methionine (SAM)-dependent type IV riboswitch upstream of $c g 1478$ (annotated as hypothetical protein). Together with the two riboswitch related RNA-motifs (both yybP-ykoY) [40] and seven additional transcripts putatively encoding small proteins, we ended up with 316 regions in the class "mRNA leader".

The class "antisense transcripts" comprises 543 regions (Additional file 3) and includes three sub-types (i) cisantisense RNAs (asRNA) that start in antisense orientation within an opposing CDS, (ii) transcripts antisense to a $5^{\prime}$-UTR, starting within $100 \mathrm{nt}$ from the $5^{\prime}$-end of an opposing CDS (as5'-UTR) and (iii) transcripts antisense to a 3 '-UTR, starting within 60 nt from the 3 '-end of an opposing CDS (as3'-UTR). This analysis defined 464 sRNA regions as asRNAs, 63 as as $5^{\prime}$-UTRs, and 16 as as3' -UTRs. Eight as5' -UTRs were also counted as3'UTRs and 48 mRNA leader were also counted as5'-UTRs due to special arrangements of CDS. All remaining 262 regions were designated as trans-encoded sRNAs (Additional file 4). For preparation of an updated

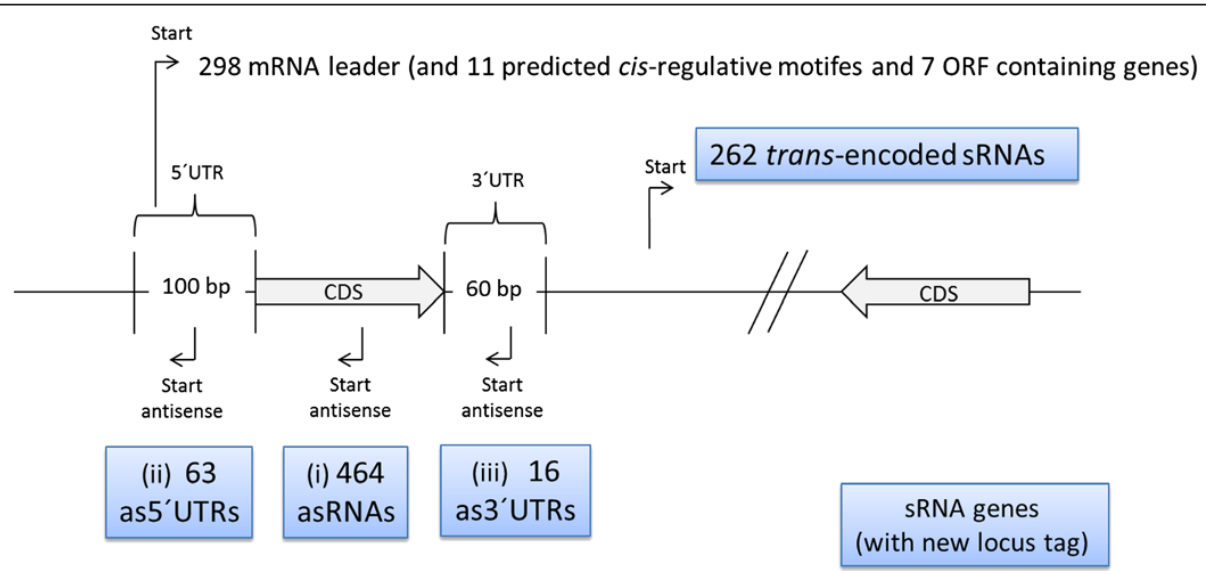

Figure 3 Classification of potential sRNA genes by their positions relative to annotated protein-coding sequences. Transcripts with a downstream CDS in less than 100 nt distance from their 3'-ends were designated as "mRNA leader". The class also includes riboswitches and ORF containing transcripts already predicted and stored in the Rfam database [9]. The class "antisense transcripts" comprises three sub-types (i) cisantisense RNAs (asRNA) that start within a CDS, (ii) transcripts antisense to a 5'-UTR, starting within 100 nt from the 5'-end of a CDS (as5'-UTR) and (iii) transcripts antisense to a 3'-UTR, starting within $60 \mathrm{nt}$ from the $3^{\prime}$-end of a CDS (as3'-UTR). All remaining intergenic transcripts were categorized as trans-encoded sRNAs. 
C. glutamicum genome annotation, only antisense transcripts and trans-encoded RNAs were assigned with locus-tags. In the new nomenclature the locus tag of each CDS will be extended by a trailing zero (e.g. old: cg0001; new: cgb_00010). The last digit of the number is used to number novel features in between of old features.

\section{Detection of small C. glutamicum genes encoding small proteins}

Some of the sRNAs might actually represent mRNAs and encode small proteins. We applied the "ORFfinder" online tool to extract ORFs from sequences in multiple FASTA format and subsequently searched for ribosomal binding sites (RBSs) upstream of the extracted ORF sequences with RBSfinder [41] using a window size of $15 \mathrm{bp}$ and the standard RBS settings. Only ORFs with a minimum length of 15 amino acid residues showing either a RBS (4) or leaderless transcripts (4) were taken into account, and in total eight small mRNAs were retrieved (Additional file 5). Next, we searched for conservation of these peptide sequences in other bacterial genomes using the TBlastX algorithm at the NCBI web portal (http://blast.ncbi.nlm.nih.gov/Blast.cgi) and found conservation in seven cases (Additional file 5). Similar small proteins with E-values less than $10^{-4}$ were found in other Corynebacterium species and also outside Corynebacteria. More widely conserved proteins comprise the well conserved peptide-tag encoded by the tmRNA that was identified with 12 amino acids length (AEKSQRDYALAA) in C. glutamicum. Beside the peptide encoded by tmRNA, only one other peptide, $c g b \_08775$ (cg4014), was detected to be conserved in species beyond Corynebacterium. The smallest of all conserved peptides with 15 amino acids length was found to be $c g b \_14345$ ( $\left.c g 4016\right)$. This peptide is already known as valine-containing leader peptide in front of the $i l v B N C$ operon [42]. Further putative leader peptides of attenuator structures were detected at cgb_33575 (cg4012) located upstream of $\operatorname{trp} E$ of the tryptophan operon, at cgb_03035 (cg4015) in front of the leuA gene (cg0303), encoding isopropylmalate synthase, the first step in leucine biosynthesis, and at aroF (cg1129). Supporting their functional assignment is the occurrence of three consecutive tryptophan residues in the putative leader peptide upstream of the tryptophan operon, four consecutive leucine codons in the presumed leuA leader peptide and the amino acids phenylalanine-tyrosinephenylalanine in the case of the aroF leader peptide.

\section{Analysis of cis-antisense RNA genes, located within protein-coding genes}

In our study, more than half of the sRNA regions (543 of 807) fall into the class "antisense transcripts" (Figure 3,
Additional file 3). For the sub-type of asRNAs (464), which are located directly opposite to a CDS, the mean length was calculated to be $55 \mathrm{nt}$. This very small size particularly for asRNAs is shown in a box plot diagram (Additional file 6). The asRNAs are distributed to 409 different CDSs with 44 CDS having more than one antisense transcript. It is noteworthy that antisense transcription is not only observed at the $5^{\prime}$-ends of the corresponding CDS, but often also at the 3 '-ends or in the middle of a coding region. To correlate the functions of encoded proteins with observed asRNAs, the respective proteins were classified according to the eggNOG functional classification system [43]. Thereby, 264 of 464 asRNAs match to CDS with eggNOG classification (Figure 4). In case of the remaining 200 asRNA, either no category or categories with poor characterization were retrieved. A normal distribution of 464 asRNAs over all currently annotated CDS would result in about $15 \%$ of the genes of each eggNOG class to contain a asRNA. We considered classes containing a asRNA in more than $20 \%$ or less than $10 \%$ of the members of the class as over- or underrepresented. The lowest proportions of asRNAs were observed for genes within the classes' cell cycle control/cell division (" $\mathrm{D}$ ", $0 \%$ ), coenzyme transport and metabolism ("H", 1\%), transcription ("K", 7\%), and inorganic transport and metabolism ("P", 9\%). On the other hand, we observed a higher proportion of asRNAs within genes from the functional class cell envelope biogenesis ("M", 35\%). Moreover, a higher proportion of genes which are involved in secretion processes ("U", 32\%) seem to have transcription in antisense direction (Figure 4). Among the genes involved in protein secretion there were both protein secretion mechanisms represented, with $\sec Y$ (cg0647) encoding a preprotein translocase subunit of the Sec secretion system and tatC (cg1684) encoding a twin-arginine (Tat) secretion translocase subunit. The tatC antisense RNA (cgb_16835) is apparently transcribed from a SigAdependent promoter, whereat the sec $Y$ asRNA (cgb_06475) seems to be transcribed from a SigH-controlled promoter.

In this context, we tested also the proportions of asRNAs at genes encoding transmembrane helices or signal peptides for secretion. By bioinformatic search 165 of 464 asRNAs $(\sim 35 \%)$ were detected at the corresponding genes. Statistically, this proportion is not significantly different from a normal distribution since 974 genes ( $\sim 32 \%$ of all annotated C. glutamicum genes) encode a signal peptide for secretion or at least one transmembrane helix.

The occurrence of asRNAs includes also two of the 13 two-component regulatory systems in C. glutamicum, namely the sensory histidine kinase genes cgtS4 (cg0483) and cgtS6 (cg3060) that sense a specific environmental stimulus at the membrane and the corresponding 


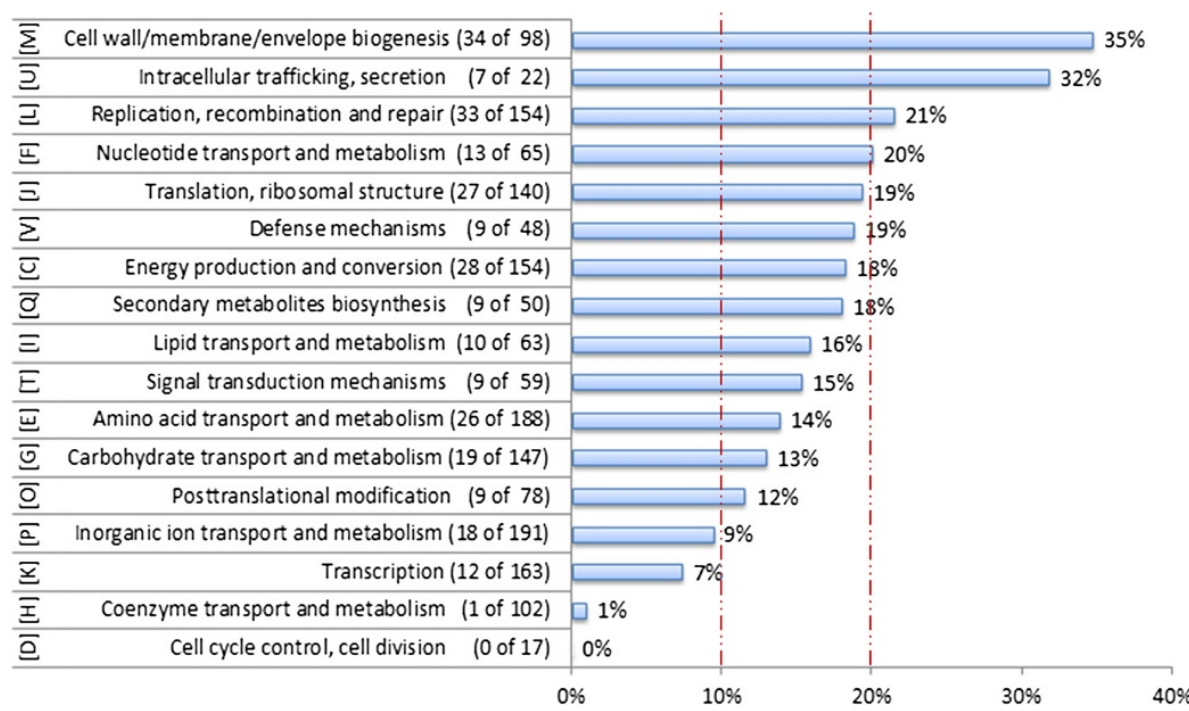

Figure 4 Functional classification of $C$. glutamicum genes having cis-antisense RNAs (asRNAs) according to eggNOG. Occurrences and frequencies of asRNAs at genes with assigned eggNOG [43] classes (264 of 464) are shown. Classes with cis-antisense RNA frequencies between $10 \%$ and $20 \%$ reflect a normal distribution. Red lines represent limits for classes that are underrepresented $(<10 \%)$ or overrepresented $(>20 \%)$.

response regulators genes cgtR4 (cg2888) and cgtR6 (cg3061) that mediate the transcriptional regulation by binding to operators [44]. The cgtSR4 genes are involved in phosphate starvation [45] and cgtR4 seems to be essential [46]. Further asRNAs are located opposite to transcriptional regulator genes (within eggNOG class $\mathrm{K}$ ) such as cysR (cg0156) and sufR (cg1756) which are involved in assimilatory sulphate reduction [47] and thiol-oxidative stress defense [48], respectively. Beside this, we detected asRNAs at the $a c n R$ gene (cg1738) [49] and other members of the TetR family (cg2686; cg1308). The following transcriptional regulators were also identified to have antisense transcription, SugR (cg2115) as regulator of the PEP:sugar phosphotransferase system genes [50,51], NdrR (cg2112) the regulator of deoxyribonucleotide reduction [52], PcaO (cg2627) the transcriptional activator of the ketoadipate metabolism genes [53], FarR (cg3202) a transcriptional regulator involved in nitrogen metabolism [54], and two members of the HTH_3-family (cg1392; cg2040).

\section{Bioinformatic analysis of sequence and structural conservation of trans-encoded sRNAs in C. glutamicum ATCC 13032}

In bacteria sRNAs often have characteristic structures that are conserved stronger in evolution than their primary sequences. Hence, structure conservation analysis is integrated in a number of sRNA prediction tools. In our approach, we used the RNAz tool [20] to detect secondary structure conservation in a multiple genome alignment between the closely related species of C. glutamicum ATCC 13032, C. efficiens YS-314 and C. diphtheriae
NCTC 13129. RNAz predictions made under stringent conditions $(\mathrm{p} \geq 0.9)$ overlap with 45 trans-encoded sRNA genes detected with RNA-Seq (Table 2). Moreover, the sequencing results were compared with a less stringent set of RNAz predictions $(\mathrm{p} \geq 0.5)$ which resulted in 77 of 262 trans-encoded sRNAs matching to loci predicted by RNAz ( 30\%) (marked in Additional file 4). Hereby, all three housekeeping RNAs, tmRNA (cgb_09183), M1 RNA (cgb_24535), and 4.5S RNA (cgb_02933) were predicted at positions very similar to those of the Rfam database entries that were calculated by covariance models [9]. This was the case also for 6C RNA (cgb_03605), which is known to be present in many Actinomycetales genera [55]. The 6C RNA was named from its two stem-loops, each typically containing six cytosine $(\mathrm{C})$ residues. Interestingly, in C. glutamicum the 6C RNA has two stretches of eight cytosines. However, the function of these cytosine homopolymers is not known and therefore the relevance of this difference is unclear.

Experimental validation of three trans-encoded sRNAs by Northern blotting of stress-specific RNA samples and detailed structure analysis

For the total set of detected trans-encoded sRNAs we retrieved a mean length of $90 \mathrm{nt}$ (Additional file 6). To validate the sequencing approach, three sRNAs were selected and subsequently analyzed by Northern blotting: the highly conserved 6C RNA (cgb_03605) (Figure 5a) and two sRNAs with high read counts at their TSS (> 1000). We chose cgb_00105 (upstream of cg0010) as an example of a sRNA with strong secondary structure conservation as shown above (Table 2; Figure 5b) and 
Table 2 Trans-encoded sRNA genes with overlapping RNAz-prediction $(p \geq 0.9)$ and their prediction details New locus tag Strand Sequencing start Sequencing stop Adjacent genes RNAz Prediction start RNAz Prediction end

\begin{tabular}{|c|c|c|c|c|c|c|c|}
\hline cgb_00105 & - & 10053 & 9921 & cg0010(-)/cg0012(-) & 10073 & 9921 & 0.95 \\
\hline cgb_00925 & + & 74286 & 74320 & cg0092(+)/cg0095(+) & 74297 & 74476 & 0.99 \\
\hline cgb_03505 & - & 307582 & 307548 & cg0350(-)/cg0352(-) & 307558 & 307474 & 0.95 \\
\hline${ }^{a}$ cgb_03605 & + & 314679 & 314787 & cg0360(-)/cg0362(+) & 314611 & 314792 & 0.99 \\
\hline cgb_03995 & - & 346945 & 346882 & cg0399(-)/cg0400(-) & 346922 & 346590 & 0.96 \\
\hline cgb_05085 & + & 452359 & 452408 & cg0508(-)/cg0510(+) & 452321 & 452622 & 0.99 \\
\hline cgb_05716 & + & 509744 & 509990 & cg0571(+)/cg0572(+) & 509724 & 509981 & 0.99 \\
\hline cgb_05756 & + & 512702 & 512814 & cg0575(+)/cg0576(+) & 512744 & 512906 & 0.99 \\
\hline cgb_08496 & + & 782757 & 782836 & cg0849(+)/cg0850(+) & 782647 & 782889 & 0.99 \\
\hline cgb_08785 & - & 807467 & 807331 & cg0878(-)/cg0879(+) & 807563 & 807292 & 0.92 \\
\hline cgb_09095 & - & 842812 & 842715 & cg0909(-)/cg0910(-) & 842945 & 842791 & 0.97 \\
\hline cgb_09097 & - & 842983 & 842911 & cg0909(-)/cg0910(-) & 842945 & 842791 & 0.97 \\
\hline bcgb_09185 & + & 848500 & 848922 & cg0918(+)/cg0919(+) & 848444 & 848993 & 0.90 \\
\hline cgb_09483 & + & 878863 & 878996 & cg0948(-)/cg0949(+) & 878852 & 879125 & 0.99 \\
\hline cgb_13305 & - & 1237440 & 1237333 & cg1330(+)/cg1332(-) & 1237507 & 1237208 & 0.90 \\
\hline cgb_14495 & + & 1351975 & 1352041 & cg1449(+)/cg1451(+) & 1351833 & 1352246 & 0.99 \\
\hline cgb_17355 & - & 1626662 & 1626596 & cg1735(-)/cg1736(-) & 1626885 & 1626583 & 0.95 \\
\hline$c g b \_17735$ & + & 1665705 & 1665791 & cg1773(-)/cg1774(+) & 1665657 & 1665835 & 0.99 \\
\hline cgb_17805 & - & 1672717 & 1672673 & cg1780(+)/cg1781(-) & 1672744 & 1672565 & 0.92 \\
\hline cgb_18405 & - & 1734383 & 1734304 & $\operatorname{cg} 1840(-) / c g 1841(-)$ & 1734440 & 1734264 & 0.99 \\
\hline cgb_18415 & - & 1736390 & 1736347 & cg1841(-)/cg1842(+) & 1736478 & 1736333 & 0.96 \\
\hline cgb_21516 & - & 2039580 & 2039466 & cg2151(-)/cg2152(-) & 2039656 & 2039493 & 0.98 \\
\hline$c g b \_21673$ & - & 2055867 & 2055764 & cg2167(-)/cg2168(-) & 2055929 & 2055750 & 0.99 \\
\hline cgb_22185 & - & 2108839 & 2108800 & cg2218(-)/cg2221(-) & 2109027 & 2108748 & 0.98 \\
\hline cgb_22215 & - & 2110108 & 2109924 & cg2221(-)/cg2222(-) & 2110102 & 2109913 & 0.99 \\
\hline cgb_22285 & - & 2116294 & 2116236 & cg2228(-)/cg2229(-) & 2116297 & 2115998 & 0.96 \\
\hline cgb_22405 & - & 2124418 & 2124384 & cg2240(+)/cg2241(-) & 2124504 & 2124285 & 0.92 \\
\hline$c g b \_23783$ & - & 2267593 & 2267546 & cg2378(-)/cg2380(-) & 2267720 & 2267551 & 0.93 \\
\hline cgb_24455 & - & 2331195 & 2331116 & cg2445(-)/cg2446(-) & 2331257 & 2331109 & 0.99 \\
\hline${ }^{c} c g b \_24535$ & - & 2343003 & 2342592 & cg2453(-)/cg2455(-) & 2343050 & 2342650 & 0.95 \\
\hline$c g b \_24775$ & - & 2362678 & 2362640 & cg2477(-)/cg2478(-) & 2362704 & 2362548 & 0.94 \\
\hline cgb_25636 & + & 2447380 & 2447441 & cg2563(+)/cg2564(-) & 2447231 & 2447490 & 0.94 \\
\hline cgb_25955 & - & 2476453 & 2476419 & cg2595(-)/cg2597(-) & 2476484 & 2476295 & 0.92 \\
\hline cgb_26475 & - & 2530082 & 2530004 & cg2647(-)/cg2648(+) & 2530150 & 2529972 & 0.91 \\
\hline cgb_28315 & + & 2693243 & 2693292 & cg2831(-)/cg2833(+) & 2692958 & 2693349 & 0.99 \\
\hline cgb_28685 & - & 2730160 & 2730126 & cg2868(+)/cg2869(-) & 2730432 & 2730065 & 0.97 \\
\hline cgb_29606 & - & 2816731 & 2816535 & cg2960(+)/cg2962(-) & 2816711 & 2816532 & 0.99 \\
\hline cgb_30116 & - & 2863994 & 2863960 & cg3011(-)/cg3012(-) & 2864045 & 2863846 & 0.95 \\
\hline cgb_30685 & - & 2928726 & 2928634 & cg3068(-)/cg3069(-) & 2928804 & 2928585 & 0.99 \\
\hline cgb_31375 & - & 2997519 & 2997485 & cg3137(-)/cg3138(+) & 2997679 & 2997500 & 0.94 \\
\hline cgb_31785 & - & 3041252 & 3041154 & cg3178(-)/cg3179(-) & 3041427 & 3041124 & 0.97 \\
\hline
\end{tabular}


Table 2 Trans-encoded sRNA genes with overlapping RNAz- prediction ( $\mathbf{p} \geq 0.9)$ and their prediction details (Continued)

\begin{tabular}{llllllll}
\hline cgb_33045 & - & 3156331 & 3156287 & $c g 3304(-) / c g 3306(-)$ & 3156621 & 3156254 & 0.91 \\
cgb_33325 & + & 3179928 & 3180066 & $c g 3332(-) / c g 3334(+)$ & 3179728 & 3180003 & 0.99 \\
cgb_34325 & - & 3282122 & 3282086 & cg3432(-)/cg3434(+) & 3282124 & 3281877 & 0.95 \\
\hline
\end{tabular}

a RF01066; 6C.

${ }^{\mathrm{b}} \mathrm{RF} 00023 ;$ tmRNA.

${ }^{\mathrm{c} R F 00010 ;}$ RNaseP.

cgb_20715 (downstream of cg2071) as an example lacking secondary structure conservation (Figure 5c). Secondary structures of all presented sRNAs were determined by minimum free energy folding and RNA shape analysis [56] which achieved high shape probabilities $(\sim 90 \%)$ in all cases, respectively. Here, the total RNAs obtained from different growth conditions were analyzed separately to monitor stress-specific transcription patterns. The sizes of these sRNAs determined by Northern blot are as follows: $\sim 100 \mathrm{nt}$ for $6 \mathrm{C}$ RNA, $130 \mathrm{nt}$ for $c g b \_00105, \sim 90$ nt and $\sim 70$ nt for $c g b \_20715$. In each case, the Northern blot signal of the longest sRNA correlated well with the length determined by sequencing. Compared with the Rfam prediction, the 6C RNA had a 27 nt 5 '-extension (Figure 5a). This extended 5' -region seems to be conserved in the Corynebacterium species, since a longer 5 '-region for 6C RNA was also predicted by our RNAz approach (data not shown).

The 6C RNA showed no change in transcript abundance in the Northern blots (Figure 5a). Probably due to a regulatory mechanism, cgb_00105 appeared to be absent under heat stress conditions (Figure $5 \mathrm{~b}$ ). The sRNA cgb_20715 also does not appear to be differentially transcribed at a tested condition. Interestingly, the Northern blot revealed a shorter second band of at $\sim 70 \mathrm{nt}$, indicating a second RNA species possibly generated by RNA processing, since no additional promoter was observed in this region.

\section{Discussion}

RNA sequencing is a novel approach to characterize transcriptomes of bacteria comprehensively. This technique is especially useful for detection of novel sRNAs. Here, we present the first small RNA-Seq approach for C. glutamicum, a member of the genus Corynebacterium, which represents also a model organism for the closely related genera within the Corynebacterineae, e.g. Mycobacterium, Nocardia etc. In comparison to the knowledge of regulatory sRNAs in the class of GammaProteobacteria, especially in E. coli and Salmonella species, information about sRNAs is marginal in Actinobacteria. Until now, deep sequencing of transcriptomes in this class has only been reported for Mycobacteria $[57,58]$ and Streptomyces species [7].

Since sRNAs might be differentially transcribed under stress, a mixed sample of various conditions should ensure the transcription of as many sRNAs as possible. The isolation of small RNAs, however, yielded not only "true" sRNAs but also a lot of RNAs that are processed or in the process of degradation. Therefore, a number of filtering steps were performed on the cDNA reads achieved. Besides using a chosen cut-off for the increase in the number of read starts relative to the preceding position for calling a transcript start, the 5 '-ends were validated by promoter searches, and from these validated 5 '-ends, 3 '-ends of transcripts were determined by another chosen cut-off of ten reads. It has to be stated that these cut-offs were arbitrary and adjusted to the size of the data set.

As the next step, transcripts were classified by their relative positions to annotated protein-coding sequences (CDS). The length of $5^{\prime}$-UTRs of coding sequences is variable, zero for leaderless transcripts and especially long for genes regulated by cis-regulative elements such as riboswitches. The difficulty of UTR length definition was also reported in other studies $[59,60]$. Interestingly, we observed a short transcript length particularly for asRNAs. As expected, the class of leader mRNAs represents the longest transcripts, resulting from transcription into the adjacent CDS. This difference was not obtained for the different types of sRNAs in Sinorhizobium meliloti [60]. Generally, a shorter average size of sRNAs compared to sRNAs from other bacteria was also reported from Streptomyces coelicolor [7].

Cis-antisense sRNAs are abundant in C. glutamicum and located in $\sim 15 \%$ of all annotated protein-coding genes. High-resolution tiling arrays and RNA sequencing led to the discovery of extensive antisense transcription in several other bacteria (reviewed in $[18,61,62]$ ). In these previous studies, the reported percentage of genes within a genome which are targeted by asRNA varies up and is $>46 \%$ in Helicobacter [26]. The first asRNA in C. glutamicum was detected upstream of cg1935 [8], thereby overlapping the mRNA of the transcriptional regulator of the GntR family in antisense direction. In our study, we detected four more asRNAs which are located opposite to already known transcriptional regulatory genes and further five asRNAs at putative regulatory genes. So far, it has been investigated that asRNAs can modulate the level of transcriptional regulators, metabolic, toxic and virulence proteins or repress transposases (reviewed in [61]). For C. glutamicum, our 


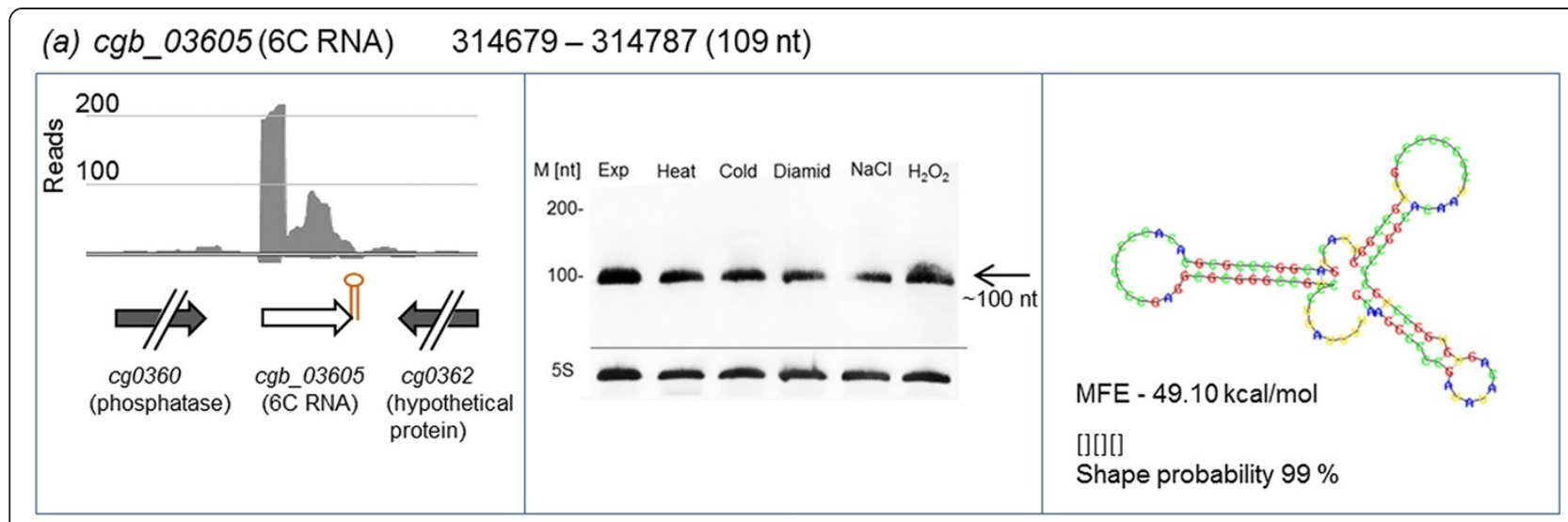

(b) cgb_00105 10053-9921(131 nt)

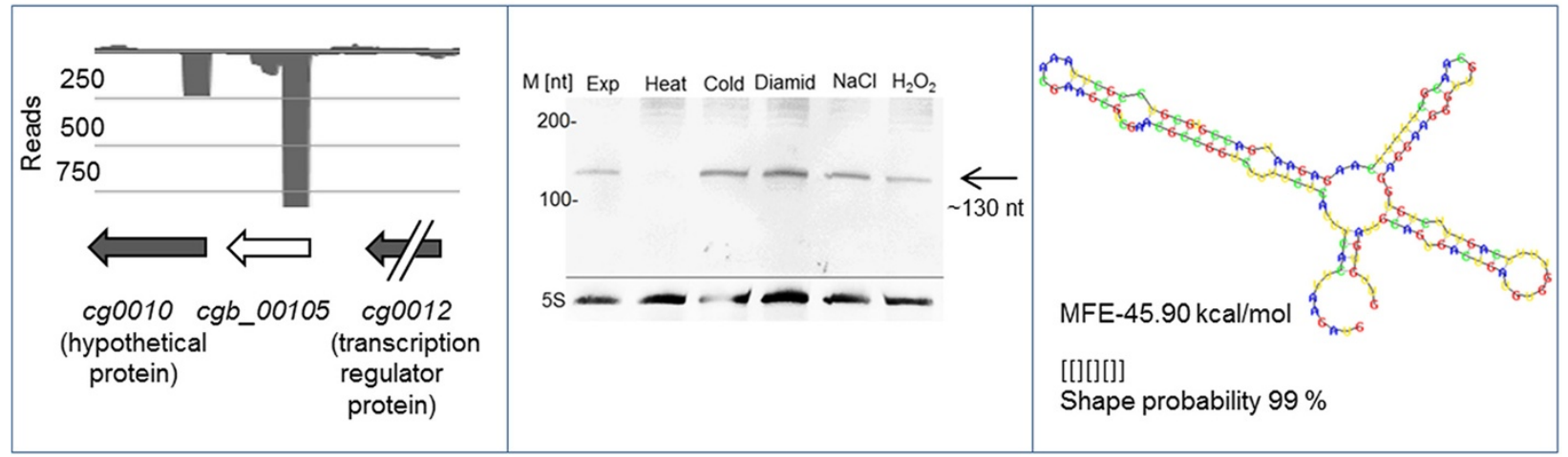

(c) $c g b \_20715$

1965663-1965755 (93 nt)

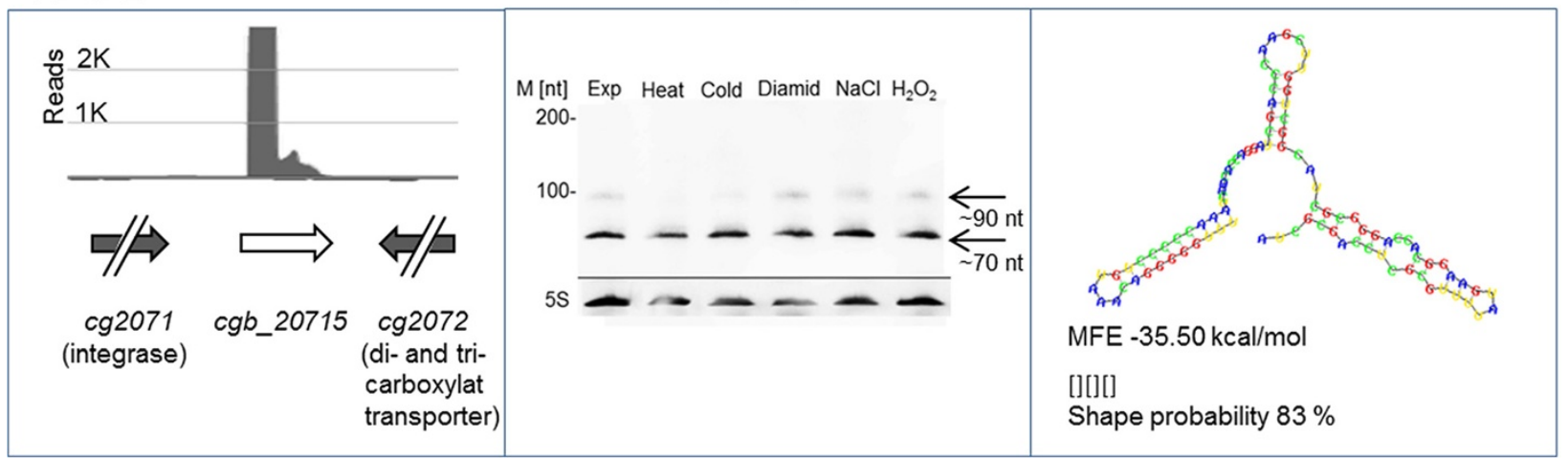

Figure $\mathbf{5}$ Secondary structure prediction and experimental validation by Northern hybridizations of three selected sRNAs. For each of the three sRNA genes, the left column shows the sequence coverage profile derived from library 1 (grey color). The $y$ - and $x$-axis represent coverage and sequence localization. Grey arrows represent flanking genes, white arrows represent the sRNAs and brown stem-loop structures represent Rho-independent terminators. The middle column displays Northern blot results of all tested conditions at time point of harvesting of C. glutamicum cells, respectively. Exp $=$ Exponential phase $\left(\mathrm{OD}_{600} 10\right)$, unstressed. Further conditions were heat, cold, diamide, $\mathrm{NaCl}$, and $\mathrm{H}_{2} \mathrm{O}_{2}$ stresses, all applied for 15 minutes at an $\mathrm{OD}_{600}$ 10. The right column presents the secondary structure with lowest minimum free energy (MFE). Structure, MFE and shape probabilities determined with RNAShapes [56]. Sequence code: blue, A; green, C; red, G; yellow, U. Validated intergenic sRNAs by Northern hybridizations are (a) cgb_03605 (6C RNA), (b) cgb_00105 and (c) cgb_20715.

analysis of antisense transcripts based on the eggNOG classification system revealed that asRNAs seem to occur frequently at genes encoding proteins with functions in cell envelope biogenesis and protein secretion processes. However, there are hundreds of short transcripts in antisense direction of coding sequences in C. glutamicum the functions of which remain to be elucidated. In general, antisense transcripts often influence RNA stability of their target mRNA either by promoting or blocking ribonucleases [63-66]. Furthermore, asRNAs 
can induce a structural change in their target mRNA that effects transcription attenuation [67]. Other studies showed that asRNA can also hinder RNA polymerase extending the transcript encoded in the opposite strand by transcription interference (reviewed in [68]) or can affect translation of the target gene by regulation of ribosome binding [69].

Further classification, especially of trans-encoded sRNAs, can be done by sequence and structural analysis. The comparison of candidates predicted by the RNAz tool [20] and by the Rfam database [9] with sequencing results, allowed us to detect sRNAs that are conserved in all bacteria (housekeeping genes), in Actinomycetales (6C RNA) or within the closely related species $C$. efficiens and C. diphtheriae. However, more than the half of sRNAs seems to be specific for $C$. glutamicum. At this point we want to note that our study did not detect two widely conserved elements known to be involved in bacterial sRNA: 6S RNA [70] and short palindromic repeat (CRISPR) loci (reviewed by [71]). Interestingly, at least one CRISPR locus has been identified in the genomes of almost all other Corynebacterium species (CRISPRdB) [72,73].

Many bioinformatic prediction tools were developed for sRNA research during the last decade. The comparison of the actual in vivo expression of sRNAs with bioinformatic prediction results often revealed only little correspondence $[7,74,75]$. Apart from the RNAz program [20] which was utilized in this study, the sRNAPredict algorithms $[21,38,76]$ are prominent bioinformatic tools which have been used in various bacterial sRNA studies. As implemented in sRNAPredict, the analysis on Rho-independent terminators is often integrated in tools for sRNA detection. However, more than $75 \%$ of the trans-encoded sRNAs detected by our sequencing approach are not followed by a Rhoindependent terminator and especially the number of asRNAs with Rho-independent terminators is marginal. A similar observation was obtained within in a search for sRNAs in Vibrio splendidus [77]. The correct termination of one sRNA (cgb_00105) at a site without an obvious terminator structure was proven by Northern blot analysis.

RNA-Seq analyses deliver an unmatched single nucleotide resolution. However, confirmatory methods are required, such as Northern blotting and are used in the present study, to look at stress-specific transcription. An example is presented with $c g b \_00105$ : under heat shock we detected no transcription of this sRNA whereas cold shock and chemically induced stresses had no influence on the amount of transcript. Heat shock condition was also observed to trigger a different transcription start site for ArnA cis-antisense RNA [8]. In the case of $c g b \_20715$, two transcripts of different length are detected in each case. Here, rather sRNA maturation or degradation by endo- or exoribonucleases is likely. For 6C RNA no change by one of the chosen stress treatments was observed. At this point, there is no hint for the function of 6C RNA in C. glutamicum. Currently, the $6 \mathrm{C}$ RNA was reported to be involved in the GlxR regulatory network in C. glutamicum [78]. GlxR is known as a global regulator of carbon source metabolism and energy conversion. In Streptomyces coelicolor, 6C RNA showed an increased transcription during sporulation [79].

In bacteria there is an additional group of transcripts, comprising RNAs that act as both, regulatory RNAs and mRNAs. RNA with dual properties is exemplified by tmRNA, which combines the features of a tRNA and an mRNA. This housekeeping RNA recycles stalled ribosomes by adding a proteolysis-inducing tag to unfinished polypeptides [80]. Our results show that the tmRNA peptide-tag in C. glutamicum corresponds well to known sequences of a wide phylogenetic spectrum [81].

Short peptides encoded within $5^{\prime}$-UTRs of mRNA sequences are known as characteristic feature in a mechanism called transcriptional attenuation. In our study, we detected attenuator transcripts at different genes and operons involved in amino acid synthesis, each encoding a suitable leader peptide. Such RNAs are also included as cis-regulatory motives in the Rfam database. Transcriptional attenuation was first described for the tryptophan (trp) operon in E. coli [82] where terminator formation is associated to a leader sequence and is influenced by the availability of $\mathrm{tRNA}^{\mathrm{Trp}}$ (RF00513). Accordingly, it has been observed for the ilvBNC operon in C. glutamicum [42] and different amino acid operons in other microorganisms (reviewed in [83]. With our analyses, we could predict further genes and operons involved in the biosynthesis of different amino acids to be regulated by the availability of uncharged tRNAs. These were detected upstream of $\operatorname{trp} E$ of the tryptophan operon, in front of the leuA gene (cg0303), encoding isopropylmalate synthase, the first step in leucine biosynthesis, and at aroF (cg1129), encoding one of the two DAHP Synthases [84] in C. glutamicum, responsible for the first step of shikimate pathway in the biosynthesis of aromatic amino acids. Since C. glutamicum is a wellknown industrial producer of amino acids, these findings might become relevant for future engineering of amino acid producer strains.

\section{Conclusions}

Our present study represents the first comprehensive screening for small RNAs in Corynebacteriaceae, a family that comprises important bacteria of industrial and medical relevance. High-throughput sequencing techniques are often applied for the search and investigation of sRNAs in bacterial genomes. Similar to sRNA studies 
in other bacteria, we detected hundreds of sRNA genes in C. glutamicum ATCC 13032. In C. glutamicum, more than half of all small RNAs genes was classified as antisense transcripts. Cis-antisense sRNA genes were detected at CDS with various functions. However, CDS specifying proteins from the functional classes 'cell envelope biogenesis' and 'secretion processes' appear to be overrepresented. Trans-encoded sRNA genes were found distributed over the entire genome and showed secondary structure conservation among corynebacteria in about 30\%. The 6C RNA, already known from other Actinomycetales genera showed strong transcription at unstressed exponential growth and all tested stress conditions. The 6S RNA, highly conserved in bacteria, was not found in C. glutamicum. Additionally, we detected riboswitches, transcriptional attenuators and other cisregulatory motives, demonstrating the potential of our study for unraveling novel regulatory processes by small RNAs in C. glutamicum.

\section{Methods}

Preparation of cDNA libraries for RNA-Sequencing Bacterial growth conditions and total RNA-isolation

C. glutamicum ATCC 13032 was grown in CGXII minimal medium at $30^{\circ} \mathrm{C}$ until exponential phase $\left(\mathrm{OD}_{600}\right.$ 10). Cells were treated in five different stress experiments by heat $\left(50^{\circ} \mathrm{C}\right)$, cold $\left(4^{\circ} \mathrm{C}\right)$, diamide $\left(\mathrm{N}, \mathrm{N}, \mathrm{N}^{\prime}, \mathrm{N}^{\prime}\right.$ tetramethylazodicarboxamide, $2 \mathrm{mM}), \mathrm{NaCl}(1.5 \mathrm{M})$, and $\mathrm{H}_{2} \mathrm{O}_{2}(0.33 \mathrm{M})$ for 15 minutes. After harvesting $2 \mathrm{~mL}$ bacterial culture, pellets were resuspended in $1 \mathrm{~mL} \mathrm{TRIzol}{ }^{\circledR}$ reagent (Life Technologies Corporation, Darmstadt, Germany) followed by ethanol precipitation. Afterwards, crude RNA samples were treated with DNase I (Roche Diagnostics, Penzberg, Germany). After purification using phenol/chloroform/isoamyl alcohol (ratio 25:24:1), RNA was precipitated with $0.3 \mathrm{M}$ sodium acetate. Purified total RNA pellets were dissolved in $50 \mu \mathrm{L}$ RNase-free $\mathrm{ddH}_{2} \mathrm{O}$. Afterwards, the purified total RNA was qualified by Agilent RNA Nano 6000 Kit on Agilent 2100 Bioanalyzer (Agilent Technologies, Böblingen, Germany).

\section{Preparation of two different cDNA libraries for sequencing}

The purified total-RNA samples were pooled in equal parts (each condition $16 \mu \mathrm{g}$ ) and precipitated for sRNAs $<250 \mathrm{nt}$ with (2.5 M sodium acetate, 25\%; PEG 8000). Afterwards, rRNAs were depleted by Ribo-Zero for Gram-Positive Bacteria (Epicentre, Madison, USA). The sRNA-pool was then divided into two samples (each $5 \mu \mathrm{g}$ ). One sample (library 1) was enriched for primary transcripts by enzymatic treatment with Terminator 5'-Phosphate-Dependent Exonuclease and RNA-5'-Polyphosphatase (both enzymes from Epicentre, Madison, USA) while the second sample was prepared as whole small transcript library (library 2). The two sequencing libraries were then prepared according to the manufacturer's instructions of TruSeq, Small RNA Kit (Illumina, San Diego, USA). Single-stranded cDNAs were created with SuperScriptII Reverse Transciptase (Life Technologies GmbH, Darmstadt, Germany). Following this, double-stranded cDNAs were generated by PCR using adapter specific primers. Afterwards, the purified libraries were quantified and qualified by Agilent High Sensitivity DNA Kit on Agilent 2100 Bioanalyzer (Agilent Technologies, Böblingen, Germany). The sequencing of the libraries was carried out at the Center for Biotechnology, Bielefeld University, utilizing the Cluster Station and the Genome Analyzer IIx (Illumina, San Diego, USA). Each sample was sequenced on one separate lane and obtained 35 bases long single reads from the $5^{\prime}$-ends. Data analysis and base calling were accomplished using the Illumina instrument software.

\section{Bioinformatics analysis \\ Read mapping and data visualization}

Reads were mapped to the C. glutamicum ATCC 13032 genome sequence [3] with SARUMAN [30] allowing for up to two errors per read. For the visualization of short read alignments, Read Explorer (Hilker et al., manuscript in preparation) was used. The Read Explorer software enables the import and visualization of a reference sequence and appropriate mapping data as so-called tracks. It is possible to scroll through the reference genome, to zoom in at each position and to look at the mapped reads at base pair level.

\section{Detection of transcription start sites}

To automatically and systematically detect TSS, the mapping data of the library 1 enriched for primary transcripts was analyzed. First, for each strand and position of the genome, all mappings starting at the given position were counted. As possible TSS all positions on a strand were taken into account that satisfied the following criteria: for a position $i$, the number of read starts $x_{i}$ on that strand at this position exceeded a background threshold $T$ and the ratio $x_{i} / x_{i-1}$ at this position had to exceed a threshold $R$. After manual inspection of TSS, $T$ was set to 19 and $R$ to 5 as these parameters were found to result in a good signal to noise ratio.

\section{Promoter search using primary 5'-end data}

Relevant stacks for promoter search were obtained from the primary transcript enriched sequencing library. For promoter search, the tool Improbizer [31,32]) was trained with 158 published sequences from SigA binding sites [33] and $45 \mathrm{SigH}$ binding sites [34], respectively. In both cases, -10 and -35 regions were correctly identified by the expectation maximization (EM) algorithm. For determination of the background significance score, 
control runs were performed as suggested by Improbizer. This score (mean) was used as threshold in the Improbizer runs. In each run, upstream sequences of sequencing stacks were simultaneously tested with the training set at the ratio of 1:10 which showed almost no influence on the motif search and scoring. If the -10 and -35 region motif score above the threshold and exhibit a spacer length between 16-20 bp, the test sequences were signed as TSS with indicated promoter. Since well-conserved -35-regions occur seldom in C. glutamicum, SigA promoters are also indicated if the well conserved extended -10 region is calculated greater than or equal the maximum of determined background significance scores, regardless of the score of the poorly conserved -35 region.

If more than one stack with indicated promoter occurred within 3 following nucleotides (278 instances), the genomic position with the strongest relative read count was selected as TSS.

\section{Rho-independent terminator search}

The search for Rho-independent terminators in C. glutamicum ATCC 13032 was performed with the tool TransTermHP [39] at standard settings. Only hits with a confidence level $>0.75$ were rated as Rho-independent terminators. Afterwards, terminator hits were compared with data from sequencing by search for matches within 60 nt around the assumed 3'-ends of sRNA regions.

\section{ORF and RBS prediction}

ORF search was set at a minimum protein length of 48 $\mathrm{nt}$, which is known from the leader peptide of $i l v B$ transcriptional attenuator [42], up to $249 \mathrm{nt}$. The search was performed with the online tool ORFfinder by application of the following start codons: AUG, GUG, UUG and stop codons: UAA, UAG, UGA (Uhmin, Osaka University http://www.gen-info.osaka-u.ac.jp). In every case of a predicted ORF, except for leaderless transcripts, we looked for ribosome-binding sites using RBSfinder [41] applying a window size of $15 \mathrm{bp}$ and the standard RBS settings (AGGAG).

\section{Prediction of secondary structure conservation}

At first, a whole genome alignment of C. glutamicum ATCC 13032, C. efficiens YS-314 and C. diphteriae NCTC 13129 was created by MAUVE [85]. The search for conserved secondary structures was then performed with RNAz [20] in five different window sizes between 100-200 nt with a step size of 40 nt. According to Washietl and coworkers (2005) we minimized false positives by application of a RNA-class probability $\mathrm{p} \geq 0.5$ of the binary classification support vector machine (SVM), simultaneous with a mean pairwise identity (M.P.I.) > $60 \%$. Afterwards, accurate tRNA and rRNA predictions were excluded yielding in 1730 hits, some of them overlapping each other. Overlapping predictions were combined and maximum RNA-class probabilities of the combined predictions were recorded, ending up with 601 loci. In total, 339 predictions showed a more stringent value of $\mathrm{p} \geq 0.9$.

\section{Other tools and software}

RNA secondary structure analysis was performed with RNAShapes [56]. Rfam database hits for C. glutamicum ATCC 13032 genome were taken into account at bits scores $>90$. WebLogos were created as frequency plots with the online-tool at http://weblogo.berkeley.edu. All data tables were processed with Microsoft Excel 2010, box plot diagrams were created with Origin 8.5Pro.

\section{Northern blot}

Northern Blot analysis was performed with the total RNA isolated with TRIzol $^{\oplus}$ reagent (Life Technologies GmbH, Darmstadt, Germany) obtained from different growth conditions as described above. For detection of transcripts, digoxigenin (DIG)-labeled RNA probes were produced as described in [86]. The RNA probes were synthesized with primers listed in the Additional file 7.

\section{Additional files}

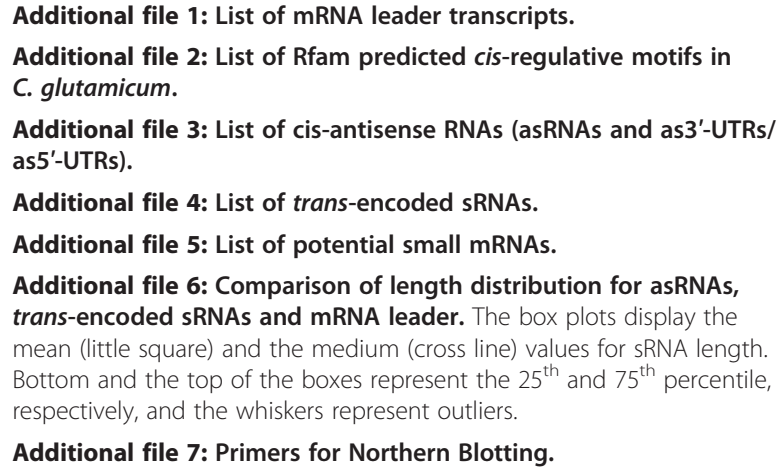

\section{Abbreviations}

RNA-Seq: High-throughput cDNA sequencing; TSS: Transcription start site(s); UTR: Untranslated region, RBS, ribosome binding site(s); bp: base pair(s); nt: nucleotide(s); OD: Optical density; $\times$ g: Times gravity; PCl: Phenolchloroform isoamyl alcohol; PEG: Polyethylene glycol; qRT-PCR: quantitative real-time polymerase-chain-reaction; rpm: rounds per minute.

\section{Competing interests}

The authors declare that they have no competing interests.

\section{Authors' contributions}

AM carried out the experimental and data analysis part, respectively, of the study, and drafted the manuscript. KPS gave assistance with analysis of RNA Seq data. CR performed the bioinformatic analysis of raw RNA-Seq data. AN and AP helped to finalize the manuscript and JK coordinated the study. All authors read and approved the manuscript.

\section{Acknowledgements}

The authors thank F. Naberhaus and I. Wilms (Ruhr-Universität Bochum) for supporting us in the technique of sRNA analysis by Northern blotting. 
Furthermore, we thank K. Gkogkoglou and R. Giegerich (Practical computer science, Bielefeld University) for assistance with RNAz prediction. We acknowledge R. Hilker and A. Goesmann (Computational genomics, Bielefeld University) for supply of unpublished software.

We additionally acknowledge grants from the Federal Ministry of Education and Research (BMBF, grants $0315585 \mathrm{~J}$ and 0316017A), as well as from the Federal State of North Rhine-Westphalia for the "Technology Platform PolyOmics" (w0805wb005).

\section{Author details}

${ }^{1}$ Microbial Genomics and Biotechnology, Center for Biotechnology, Bielefeld University, Universitätsstraße 27, 33615, Bielefeld, Germany. ${ }^{2}$ Senior Research Group Genome Research of Industrial Microorganisms, Center for Biotechnology, Bielefeld University, Universitätsstraße 27, 33615, Bielefeld, Germany. ${ }^{3}$ Technology Platform Genomics, Center for Biotechnology, Bielefeld University, Universitätsstraße 27, 33615, Bielefeld, Germany.

Received: 17 July 2013 Accepted: 25 September 2013 Published: 19 October 2013

\section{References}

1. Gopinath V, Murali A, Dhar KS, Nampoothiri KM: Corynebacterium glutamicum as a potent biocatalyst for the bioconversion of pentose sugars to value-added products. Appl Microbiol Biotechnol 2012, 93:95-106.

2. Hermann T: Industrial production of amino acids by coryneform bacteria. J Biotechnol 2003, 104:155-172.

3. Kalinowski J, Bathe B, Bartels D, Bischoff N, Bott M, Burkovski A, Dusch N, Eggeling L, Eikmanns BJ, Gaigalat L, Goesmann A, Hartmann M, Huthmacher K, Krämer R, Linke B, McHardy AC, Meyer F, Möckel B, Pfefferle W, Pühler A, Rey DA, Rückert C, Rupp O, Sahm H, Wendisch VF, Wiegräbe I, Tauch A: The complete Corynebacterium glutamicum ATCC 13032 genome sequence and its impact on the production of L-aspartate-derived amino acids and vitamins. Journal of biotechnology 2003, 104:5-25.

4. Ikeda M, Nakagawa S: The Corynebacterium glutamicum genome: features and impacts on biotechnological processes. Appl Microbiol Biotechnol 2003, 62:99-109.

5. Brinkrolf $K$, Brune I, Tauch A: The transcriptional regulatory network of the amino acid producer Corynebacterium glutamicum. J Biotechnol 2007, 129:191-211.

6. Pauling J, Röttger R, Tauch A, Azevedo V, Baumbach J: CoryneRegNet 6.0Updated database content, new analysis methods and novel features focusing on community demands. Nucleic Acids Res 2012, 40:D610-D614.

7. Vockenhuber M, Sharma CM, Statt MG, Schmidt D, Xu Z, Dietrich S, Liesegang $\mathrm{H}$, Mathews DH, Suess B: Deep sequencing-based identification of small non-coding RNAs in Streptomyces coelicolor. RNA Biol 2011, 8:468-477.

8. Zemanová M, Kaderábková P, Pátek M, Knoppová M, Silar R, Nesvera J: Chromosomally encoded small antisense RNA in Corynebacterium glutamicum. FEMS Microbiol Lett 2008, 279:195-201.

9. Burge SW, Daub J, Eberhardt R, Tate J, Barquist L, Nawrocki EP, Eddy SR, Gardner PP, Bateman A: Rfam 11.0: 10 years of RNA families. Nucleic acids research 2012, 41:D226.

10. Kin T, Yamada K, Terai G, Okida H, Yoshinari Y, Ono Y, Kojima A, Kimura Y, Komori T, Asai K: fRNAdb: a platform for mining/annotating functional RNA candidates from non-coding RNA sequences. Nucleic Acids Res 2007, 35:D145-D148.

11. Pischimarov J, Kuenne C, Billion A, Hemberger J, Cemič F, Chakraborty T, Hain T: sRNAdb: a small non-coding RNA database for gram-positive bacteria. BMC Genomics 2012, 13:384

12. Kortmann J, Narberhaus F: Bacterial RNA thermometers: molecular zippers and switches. Nat Rev Microbiol 2012, 10:255-265.

13. Serganov A, Patel DJ: Molecular recognition and function of riboswitches. Curr Opin Struct Biol 2012, 22:279-286.

14. Storz G, Vogel J, Wassarman KM: Regulation by small RNAs in bacteria: expanding frontiers. Molecular cell 2011, 43:880-891.

15. Sorek R, Cossart P: Prokaryotic transcriptomics: a new view on regulation, physiology and pathogenicity. Nat Rev Genet 2010, 11:9-16.

16. Waters L, Storz G: Regulatory RNAs in bacteria. Cell 2009, 136:615-628.

17. Wassarman KM: 6S RNA: a small RNA regulator of transcription. Curr Opin Microbiol 2007, 10:164-168
18. Lasa I, Toledo-Arana A, Dobin A, Villanueva M, Los Mozos IR D, Vergara-Irigaray M, Segura V, Fagegaltier D, Penades JR, Valle J, Solano C, Gingeras TR: Genome-wide antisense transcription drives mRNA processing in bacteria. Proc Natl Acad Sci 2011, 108:20172-20177.

19. Livny J, Waldor M: Identification of small RNAs in diverse bacterial species. Curr Opin Microbiol 2007, 10:96-101.

20. Washietl S, Hofacker IL, Stadler PF: Fast and reliable prediction of noncoding RNAs. Proc Natl Acad Sci U S A 2005, 102:2454-2459.

21. Livny J, Fogel MA, Davis BM, Waldor MK: sRNAPredict: an integrative computational approach to identify sRNAs in bacterial genomes. Nucleic Acids Res 2005, 33:4096-4105.

22. Vogel J, Bartels V, Tang TH, Churakov G, Slagter-Jäger JG, Hüttenhofer A, Wagner EGH: RNomics in Escherichia coli detects new sRNA species and indicates parallel transcriptional output in bacteria. Nucleic Acids Res 2003, 31:6435-6443.

23. Sharma CM, Vogel J: Experimental approaches for the discovery and characterization of regulatory small RNA. Curr Opin Microbiol 2009, 12:536-546.

24. Sittka A, Lucchini S, Papenfort K, Sharma C, Rolle K, Binnewies T, Hinton J, Vogel J: Deep sequencing analysis of small noncoding RNA and mRNA targets of the global post-transcriptional regulator, $\mathrm{Hfq}$. PLoS Genet 2008, 4(8):

25. Liu JM, Camilli A: Discovery of bacterial sRNAs by high-throughput sequencing. Methods in molecular biology (Clifton, N.J.) 2011, 733:63-79.

26. Sharma CM, Hoffmann S, Darfeuille F, Reignier J, Findeiß S, Sittka A, Chabas S, Reiche K, Hackermüller J, Reinhardt R, Stadler PF, Vogel J: The primary transcriptome of the major human pathogen Helicobacter pylori. Nature 2010, 464:250-255.

27. Wang Z, Gerstein M, Snyder M: RNA-Seq: a revolutionary tool for transcriptomics. Nat Rev Genet 2009, 10:57-63.

28. Underwood JG, Uzilov AV, Katzman S, Onodera CS, Mainzer JE, Mathews DH, Lowe TM, Salama SR, Haussler D: FragSeq: transcriptome-wide RNA structure probing using high-throughput sequencing. Nat Methods 2010 7:995-1001.

29. Hoe C, Raabe CA, Rozhdestvensky TS, Tang T: Bacterial sRNAs: regulation in stress. Int J Med Microbiol 2013, 303:217-229.

30. Blom J, Jakobi T, Doppmeier D, Jaenicke S, Kalinowski J, Stoye J, Goesmann A: Exact and complete short-read alignment to microbial genomes using Graphics Processing Unit programming. Bioinformatics 2011, 27:1351-1358.

31. Ao W, Gaudet J, Kent WJ, Muttumu S, Mango SE: Environmentally induced foregut remodeling by PHA-4/FoxA and DAF-12/NHR. Science 2004, 305:1743-1746.

32. Tompa M, Li N, Bailey TL, Church GM, de Moor B, Eskin E, Favorov AV, Frith MC, Fu Y, Kent WJ, Makeev VJ, Mironov AA, Noble WS, Pavesi G, Pesole G, Régnier M, Simonis N, Sinha S, Thijs G, van Helden J, Vandenbogaert M, Weng Z, Workman C, Ye C, Zhu Z: Assessing computational tools for the discovery of transcription factor binding sites. Nat Biotechnol 2005, 23:137-144.

33. Pátek $M$, Nešvera J: Sigma factors and promoters in Corynebacterium glutamicum. J Biotechnol 2011, 154:101-113.

34. Busche T, Šilar R, Pičmanová M, Pátek M, Kalinowski J: Transcriptional regulation of the operon encoding stress-responsive ECF sigma factor $\mathrm{SigH}$ and its anti-sigma factor RshA, and control of its regulatory network in Corynebacterium glutamicum. BMC Genomics 2012, 13:445.

35. Kim T, Kim H, Park J, Kim Y, Kim P, Lee H: Functional analysis of sigH expression in Corynebacterium glutamicum. Biochem Biophys Res Commun 2005, 331:1542-1547.

36. Ehira S, Teramoto H, Inui M, Yukawa H: Regulation of Corynebacterium glutamicum Heat Shock Response by the Extracytoplasmic-Function Sigma Factor SigH and Transcriptional Regulators HspR and HrcA. Journal of bacteriology 2009, 191:2964-2972.

37. Crooks GE, Hon G, Chandonia J, Brenner SE: WebLogo: a sequence logo generator. Genome Res 2004, 14:1188-1190.

38. Livny J, Teonadi H, Livny M, Waldor M: High-throughput, kingdom-wide prediction and annotation of bacterial non-coding RNAs. PLOS ONE 2008, 3(11):

39. Kingsford C, Ayanbule K, Salzberg S: Rapid, accurate, computational discovery of Rho-independent transcription terminators illuminates their relationship to DNA uptake. Genome Biol 2007, 8:R22. 
40. Barrick JE, Corbino KA, Winkler WC, Nahvi A, Mandal M, Collins J, Lee M, Roth A, Sudarsan N, Jona I, Wickiser JK, Breaker RR: New RNA motifs suggest an expanded scope for riboswitches in bacterial genetic control. Proc Natl Acad Sci U S A 2004, 101:6421-6426.

41. Suzek BE, Ermolaeva MD, Schreiber M, Salzberg SL: A probabilistic method for identifying start codons in bacterial genomes. Bioinformatics 2001, 17:1123-1130

42. Morbach S, Junger C, Sahm H, Eggeling L: Attenuation control of ilvBNC in Corynebacterium glutamicum: evidence of leader peptide formation without the presence of a ribosome binding site. J Biosci Bioeng 2000, 90:501-507.

43. Jensen $L$, Julien P, Kuhn M, Von MC, Muller J, Doerks T, Bork P: eggNOG: automated construction and annotation of orthologous groups of genes. Nucleic Acids Res 2008, 36:D250-D254.

44. Kocan M, Schaffer S, Ishige T, Sorger-Herrmann U, Wendisch VF, Bott M: Two-component systems of Corynebacterium glutamicum: deletion analysis and involvement of the PhoS-PhoR system in the phosphate starvation response. J Bacteriol 2006, 188:724-732

45. Woo HM: Regulatory and metabolic aspects of the phosphate starvation response of Corynebacterium glutamicum. Germany: Ph.D. study, Universität Düsseldorf; 2010

46. Wessel M: Funktionelle Analyse des essentiellen ZweikomponentenSignaltransduktionssystems CgtSR4 aus Corynebacterium glutamicum. Dissertation: Universität Düsseldorf; 2004.

47. Rückert C, Milse J, Albersmeier A, Koch DJ, Pühler A, Kalinowski J: The dual transcriptional regulator CysR in Corynebacterium glutamicum ATCC 13032 controls a subset of genes of the McbR regulon in response to the availability of sulphide acceptor molecules. BMC Genomics 2008, 9:483.

48. Nakunst D, Larisch C, Hüser AT, Tauch A, Pühler A, Kalinowski J: The extracytoplasmic function-type sigma factor SigM of Corynebacterium glutamicum ATCC 13032 is involved in transcription of disulfide stressrelated genes. J Bacteriol 2007, 189:4696-4707.

49. Krug A, Wendisch VF, Bott M: Identification of AcnR, a TetR-type repressor of the aconitase gene acn in Corynebacterium glutamicum. J Biol Chem 2005, 280:585-595.

50. Engels V, Wendisch VF: The DeoR-type regulator SugR represses expression of ptsG in Corynebacterium glutamicum. J Bacteriol 2007, 189:2955-2966.

51. Gaigalat L, Schlüter J, Hartmann M, Mormann S, Tauch A, Pühler A Kalinowski J: The DeoR-type transcriptional regulator SugR acts as a repressor for genes encoding the phosphoenolpyruvate:sugar phosphotransferase system (PTS) in Corynebacterium glutamicum. BMC Mol Biol 2007, 8:104.

52. Jochmann N, Kurze A, Czaja LF, Brinkrolf K, Brune I, Hüser AT, Hansmeier N, Pühler A, Borovok I, Tauch A: Genetic makeup of the Corynebacterium glutamicum LexA regulon deduced from comparative transcriptomics and in vitro DNA band shift assays. Microbiology (Reading, Engl.) 2009, 155:1459-1477.

53. Zhao K, Huang Y, Chen X, Wang N, Liu S: PcaO positively regulates pcaHG of the beta-ketoadipate pathway in Corynebacterium glutamicum. J Bacteriol 2010, 192:1565-1572.

54. Hänssler E, Müller T, Jessberger N, Völzke A, Plassmeier J, Kalinowski J, Krämer R, Burkovski A: FarR, a putative regulator of amino acid metabolism in Corynebacterium glutamicum. Appl Microbiol Biotechnol 2007, 76:625-632.

55. Weinberg Z, Wang JX, Bogue J, Yang J, Corbino K, Moy RH, Breaker RR: Comparative genomics reveals 104 candidate structured RNAs from bacteria, archaea, and their metagenomes. Genome biology 2010, 11(3):R31

56. Steffen P, Voss B, Rehmsmeier M, Reeder J, Giegerich R: RNAshapes: an integrated RNA analysis package based on abstract shapes. Bioinformatics 2006, 22:500-503.

57. Arnvig KB, Comas I, Thomson NR, Houghton J, Boshoff HI, Croucher NJ, Rose G, Perkins TT, Parkhill J, Dougan G, Young DB, Bishai WR: Sequence-Based Analysis Uncovers an Abundance of Non-Coding RNA in the Total Transcriptome of Mycobacterium tuberculosis. PLoS Pathog 2011, 7:e1002342.

58. Li S, Ng PK, Qin H, Lau JK, Lau JP, Tsui SK, Chan T, Lau TC: Identification of small RNAs in Mycobacterium smegmatis using heterologous Hfq. RNA 2013, 19:74-84.
59. DiChiara JM, Contreras-Martinez LM, Livny J, Smith D, McDonough KA, Belfort M: Multiple small RNAs identified in Mycobacterium bovis BCG are also expressed in Mycobacterium tuberculosis and Mycobacterium smegmatis. Nucleic acids research 2010, 38:4067-4078.

60. Schluter JP, Reinkensmeier J, Daschkey S, Hackenberg EE, Janssen S, Janicke S, Becker J, Giegerich R, Becker A: A genome-wide survey of sRNAs in the symbiotic nitrogen-fixing alpha-proteobacterium Sinorhizobium meliloti. BMC Genomics 2010, 11:245.

61. Thomason MK, Storz G: Bacterial antisense rnas: how many are there, and what are they doing? *. Annu Rev Genet 2010, 44:167-188.

62. Mraheil MA, Billion A, Mohamed W, Mukherjee K, Kuenne C, Pischimarov J, Krawitz C, Retey J, Hartsch T, Chakraborty T, Hain T: The intracellular sRNA transcriptome of Listeria monocytogenes during growth in macrophages. Nucleic acids research 2011, 39:4235-4248.

63. Opdyke JA, Kang J, Storz G: GadY, a small-RNA regulator of acid response genes in Escherichia coli. J Bacteriol 2004, 186:6698-6705.

64. Blomberg $P$, Wagner EG, Nordström K: Control of replication of plasmid R1: the duplex between the antisense RNA, CopA, and its target, CopT, is processed specifically in vivo and in vitro by RNase III. EMBO J 1990, 9:2331-2340.

65. Gerdes K, Nielsen A, Thorsted P, Wagner EG: Mechanism of killer gene activation. Antisense RNA-dependent RNase III cleavage ensures rapid turn-over of the stable hok, srnB and pndA effector messenger RNAs. J Mol Biol 1992, 226:637-649.

66. Lee $\mathrm{E}$, Groisman EA: An antisense RNA that governs the expression kinetics of a multifunctional virulence gene. Mol Microbiol 2010, 76:1020-1033.

67. Stork M, Di Lorenzo M, Welch TJ, Crosa JH: Transcription termination within the iron transport-biosynthesis operon of Vibrio anguillarum requires an antisense RNA. J Bacteriol 2007, 189:3479-3488.

68. Shearwin KE, Callen BP, Egan JB: Transcriptional interference-a crash course. Trends Genet 2005, 21:339-345.

69. Hernández JA, Muro-Pastor AM, Flores E, Bes MT, Peleato ML, Fillat MF: Identification of a furA cis antisense RNA in the cyanobacterium Anabaena sp. PCC 7120. J Mol Biol 2006, 355:325-334.

70. Barrick JE, Sudarsan N, Weinberg Z, Ruzzo WL, Breaker RR: 6S RNA is a widespread regulator of eubacterial RNA polymerase that resembles an open promoter. RNA 2005, 11:774-784.

71. Marraffini LA, Sontheimer EJ: CRISPR interference: RNA-directed adaptive immunity in bacteria and archaea. Nat Rev Genet 2010, 11:181-190.

72. Grissa I, Vergnaud G, Pourcel C: The CRISPRdb database and tools to display CRISPRs and to generate dictionaries of spacers and repeats. BMC Bioinforma 2007, 8:172.

73. Trost E, Al-Dilaimi A, Papavasiliou P, Schneider J, Viehoever P, Burkovski A, Soares SC, Almeida SS, Dorella FA, Miyoshi A, Azevedo V, Schneider MP, Silva A, Santos CS, Santos LS, Sabbadini P, Dias AA, Hirata R, Mattos-Guaraldi AL, Tauch A: Comparative analysis of two complete Corynebacterium ulcerans genomes and detection of candidate virulence factors. BMC Genomics 2011, 12:383.

74. Perez N, Treviño J, Liu Z, Ho SCM, Babitzke P, Sumby P, Aziz RK: A GenomeWide Analysis of Small Regulatory RNAs in the Human Pathogen Group A Streptococcus. PLOS ONE 2009, 4:e7668.

75. Arnvig KB, Young DB: Identification of small RNAs in Mycobacterium tuberculosis. Mol Microbiol 2009, 73:397-408.

76. Livny J, Brencic A, Lory S, Waldor MK: Identification of 17 Pseudomonas aeruginosa sRNAs and prediction of sRNA-encoding genes in 10 diverse pathogens using the bioinformatic tool sRNAPredict2. Nucleic Acids Res 2006, 34:3484-3493

77. Toffano-Nioche C, Nguyen AN, Kuchly C, Ott A, Gautheret D, Bouloc P, Jaca A: Transcriptomic profiling of the oyster pathogen Vibrio splendidus opens a window on the evolutionary dynamics of the small RNA repertoire in the Vibrio genus. Rna 2012, 18:2201-2219.

78. Jungwirth B, Sala C, Kohl TA, Uplekar S, Baumbach J, Cole ST, Pühler A, Tauch A: High-resolution detection of DNA binding sites of the global transcriptional regulator GIxR in Corynebacterium glutamicum. Microbiology (Reading, Engl.) 2013, 159:12-22.

79. Swiercz JP, Hindra, Bobek J, Haiser HJ, Di Berardo C, Tjaden B, Elliot MA: Small non-coding RNAs in Streptomyces coelicolor. Nucleic Acids Res 2008, 36:7240-7251.

80. Keiler KC: Biology of trans-translation. Annu Rev Microbiol 2008, 62:133-151 
81. Zwieb C, Wower I, Wower J: Comparative sequence analysis of tmRNA. Nucleic acids research 1999, 27:2063-2071.

82. Yanofsky C: Attenuation in the control of expression of bacterial operons. Nature 1981, 289:751-758.

83. Merino E, Yanofsky C: Transcription attenuation: a highly conserved regulatory strategy used by bacteria. Trends Genet 2005, 21:260-264

84. Liu Y, Li P, Zhao K, Wang B, Jiang C, Drake HL, Liu S: Corynebacterium glutamicum contains 3-deoxy-D-arabino-heptulosonate 7-phosphate synthases that display novel biochemical features. Appl Environ Microbiol 2008, 74:5497-5503.

85. Darling AC, Mau B, Blattner FR, Perna NT: Mauve: multiple alignment of conserved genomic sequence with rearrangements. Genome research 2004, 14:1394-1403.

86. Wilms I, Overlöper A, Nowrousian M, Sharma CM, Narberhaus F: Deep sequencing uncovers numerous small RNAs on all four replicons of the plant pathogen Agrobacterium tumefaciens. RNA biology 2012, 9(4):446-457.

doi:10.1186/1471-2164-14-714

Cite this article as: Mentz et al:: Comprehensive discovery and characterization of small RNAs in Corynebacterium glutamicum ATCC 13032. BMC Genomics 2013 14:714.

\section{Submit your next manuscript to BioMed Central and take full advantage of:}

- Convenient online submission

- Thorough peer review

- No space constraints or color figure charges

- Immediate publication on acceptance

- Inclusion in PubMed, CAS, Scopus and Google Scholar

- Research which is freely available for redistribution 